- Gravity Probe B was still worthwhile. The panel gave the probe a green light, but it did not rank it against other possible missions. That was a mistake, says Cornish, because most well-conceived missions look useful when considered in isolation. "It is a waste of time to do reviews of a single mission," he says. Everitt responds that Gravity Probe B was competitively reviewed numerous times during its development, but he acknowledges that, as a fundamental physics mission, it did not go through the same review process used to select astronomy missions.

In 2008, when researchers were analysing data from the flight, Cornish served on a review panel that ranked Gravity Probe B as the lowest of eight then-active missions in terms of science per dollar. In response, NASA took the remarkable step of cutting off funding for the probe before its results were in. But Stanford University, the prime contractor on the mission, succeeded in keeping the data analysis alive. Everitt and his team raised an additional $\$ 3.73$ million from private sources,

\title{
A TWIST IN SPACE-TIME
}

Gravity Probe B confirmed key predictions of general relativity by measuring two tiny shifts in the spin axis of its gyroscopes.

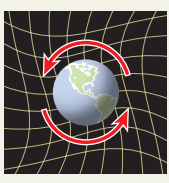

Frame-dragging effect (0.000011 degrees per year) Spinning object twists space-time.
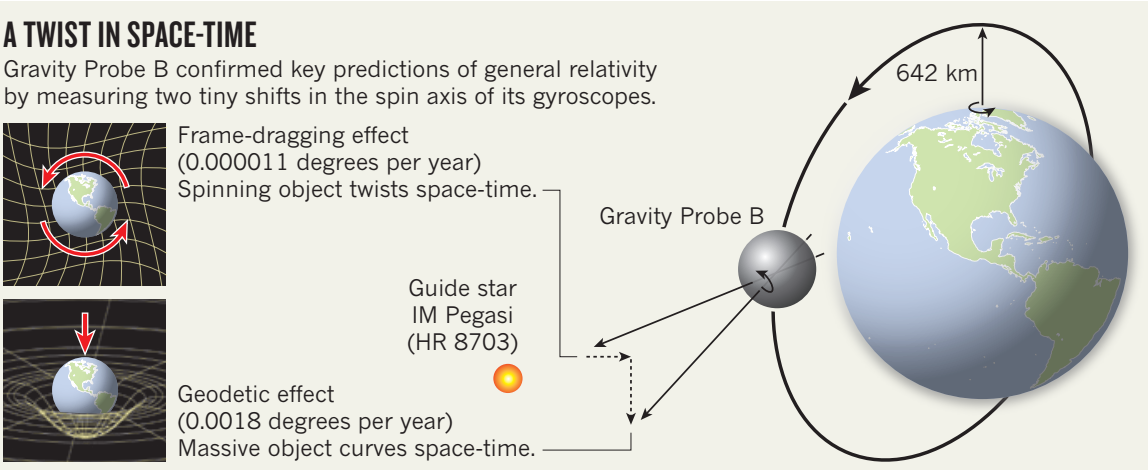

most of it from the Saudi science-funding agency King Abdulaziz City for Science and Technology (KACST), arranged through Turki Al Saud, a Saudi Arabian prince with a $\mathrm{PhD}$ from Stanford who is vice-president for research at the KACST.

Gravitation expert Bernard Schutz of the Max Planck Institute for Gravitational Physics in Potsdam, Germany, says he thinks that the mission was worthwhile because general relativity should be checked in a variety of ways. "The fact that they're getting the same thing is what we want in physics. I think it's fantastic," he says. But he laments the delays to launching a dedicated satellite that would amount to a more precise version of LAGEOS, as proposed by Ciufolini in the 1980s. "This could have been done a decade ago," Schutz says.

The Laser Relativity Satellite (LARES), on which Ciufolini is principal investigator, will finally be launched this year by the Italian Space Agency. The team intends to measure frame-dragging with $1 \%$ precision by monitoring the precession of LARES's orbit. Its cost of about $€ 4$ million (US $\$ 5.7$ million), not including launch, pales by comparison with that of Gravity Probe B.

\section{Salmonella hits US teaching labs}

\section{Wave of infections triggers investigation into biosafety practices.}

\section{BY ERIKA CHECK HAYDEN}

A spate of lab-associated Salmonella infections has swept across the United States during the past year, prompting public-health officials to examine how closely labs are following infection-prevention protocols.

"The fact that cases seem to be happening all over the country has raised the question of whether there are issues with laboratory safety and appropriate training techniques," says Mack Sewell, state epidemiologist at the New Mexico Department of Health in Santa Fe.

Between August 2010 and March this year, 73 infections due to Salmonella typhimurium, a relatively common strain of the bacterium, caused illness in people across 35 states and one death, the Centers for Disease Control and Prevention (CDC) in Atlanta, Georgia, reported on 28 April. The outbreak now seems to have ended, with the number of reported new infections dropping to the usual baseline of 0-4 per week, the CDC says.

The strain of Salmonella involved in the outbreak often causes food-borne illnesses and has been linked to past epidemics. But what sets this recent outbreak apart is that many of the illnesses have been traced back to clinical or teaching laboratories, according to the CDC report.

The agency conducted an in-depth investigation of 32 people made ill during the current outbreak, and found that $60 \%$ of them had had some connection with a microbiology laboratory in the week before their illness, compared with $2 \%$ of 64 people with other reported illnesses. The New Mexico Department of Health found that the outbreak strain was genetically identical to a commercially available strain used in some of the labs linked to ill workers or students. Furthermore, some victims of the illness reported working with Salmonella at the bench.

Laboratory-acquired infections are relatively common. One voluntary survey of 88 labs between 2002 and 2004 found that $33 \%$ had experienced at least one lab-acquired infection during that time, including six Salmonella infections (E. J. Baron and J. M. Miller Diagn. Microbiol. Infect. Dis. 60, 241-246; 2008). In 2008 , the CDC convened a panel to look into the incidence of laboratory-acquired Salmonella infections; it has not yet released a report.

But it is unusual for lab-acquired infections to crop up across the country, and the CDC is investigating how this may have happened, in part by surveying the biosafety practices of members of the American Society for Microbiology, based in Washington DC, and the Association of Public Health Laboratories in Silver Spring, Maryland.

The CDC is also concerned because some of those made ill in the outbreak, such as young children, had never visited a lab but lived with someone who worked in a lab and did not get sick. This suggests that the lab worker carried the pathogenic bacteria home on bags, clothes or other objects, says the CDC's Casey Barton Behravesh. And it raises the question of whether use of this pathogenic Salmonella strain in teaching labs is necessary.

"We are wondering whether there is a nonpathogenic or attenuated strain of Salmonella that could be used in place of this one," Barton Behravesh says.

For now, public-health officials are advising all lab workers to be more careful about observing proper biosafety procedures. Recalling a memorable lesson imparted by his own professor, Sewell suggests one way to cement the importance of those precautions: "He told us that anyone who catches something they're working with gets an automatic ' $\mathrm{F}$." - 